\title{
Resectable Colorectal Carcinoma
}

National Cancer Institute

\section{Source}

National Cancer Institute. Resectable Colorectal Carcinoma. NCI Thesaurus. Code C162257.

Colorectal carcinoma that is amenable to surgical resection. 